\title{
Using 3D Scanning to Create 4D Motion Data for Clothing Simulation
}

\author{
Christian PIRCH *, Anke KLEPSER, Simone MORLOCK \\ Hohenstein Institut für Textilinnovation gGmbH, Bönnigheim (Germany)
}

https://doi.org/10.15221/20.13

Keywords: Motion Capture, 3D Scanning, 3D simulation, 3D avatar, movement

\section{Introduction}

Digital product development processes have become increasingly established since the 1980s, especially in the clothing industry. In the last decade, technological progress has accelerated enormously and the subject of garment simulation is being discussed more and more intensively in industry and the garment industry. ${ }^{[1-4]}$ The advantages for companies that integrate digital product development into their process chain are already measurable. ${ }^{[5]}$ Through more accurate design, process and fit planning, physical prototypes can be saved, saving time and money. A clothing simulation includes the cutting data, the physical material parameters and a digital fit model. Fit models can be provided in all sizes and a simulation over the entire size range is possible. ${ }^{[6]}$ This allows the grading of a garment to be qualitatively checked and validated over several sizes. However, the quality of garment fit assessment depends on accurately measured avatars and simulated fabric behaviour ${ }^{[7]}$, combined with a deep understanding of the simulation processes of the systems used and garment engineering know-how. 3D simulation software for the virtual visualisation of garments and virtual tryon is continuously being improved. Previous statements on the fit of garments by simulation are based on a static avatar. In the project Kinematic Human Models, it has already been established that the fit control must also be carried out in different body positions. ${ }^{[8]}$ By testing the fit in typical body positions from the fields of sports, ergonomics and medicine, it is also possible to test and optimise cut constructions that have so far paid no attention to functional clothing comfort. $[9,10]$

\section{State of Art}

Movements for polygon meshes can be generated by several methods, one of the most common is rigging. In this technique, the human bone structure (rig) is reproduced in an abstract form. ${ }^{[11]}$ These bones and joints are subject to a hierarchy which affects their transformation. A bone higher in the hierarchy than the other bone is defined as parent and a lower bone as child. The points that form the avatar's polygon mesh are collected from the bones of the rig in point/vertex groups. Each point is assigned to one or more bones with a value of 0.000 to 1.000 . This value determines the extent to which a point follows the transformation of the bone. A rig consists of a forward kinematics (FK) and can be extended by an inverse kinematics (IK). The forward kinematics is a series of transformation values of the rotation axes of the bone, which together form a transformation matrix of angle values. One bone after the other is moved and the movement of the parent, angle a_2 (see figure 1) influences the position of the child. Inverse kinematics works the reverse direction, with this technique the transformation values of the parent bones are iteratively calculated from the coordinates of the child bones. With this technique there can be several possible solutions. The length of the chain of bones determines how many bones are subject to the transformation. Figure 2 shows that the chain only goes from $c$ to $b$ and therefore has a length of 2 , bone $a$ is not influenced by this.

\footnotetext{
* c.pirch@hohenstein.de
} 

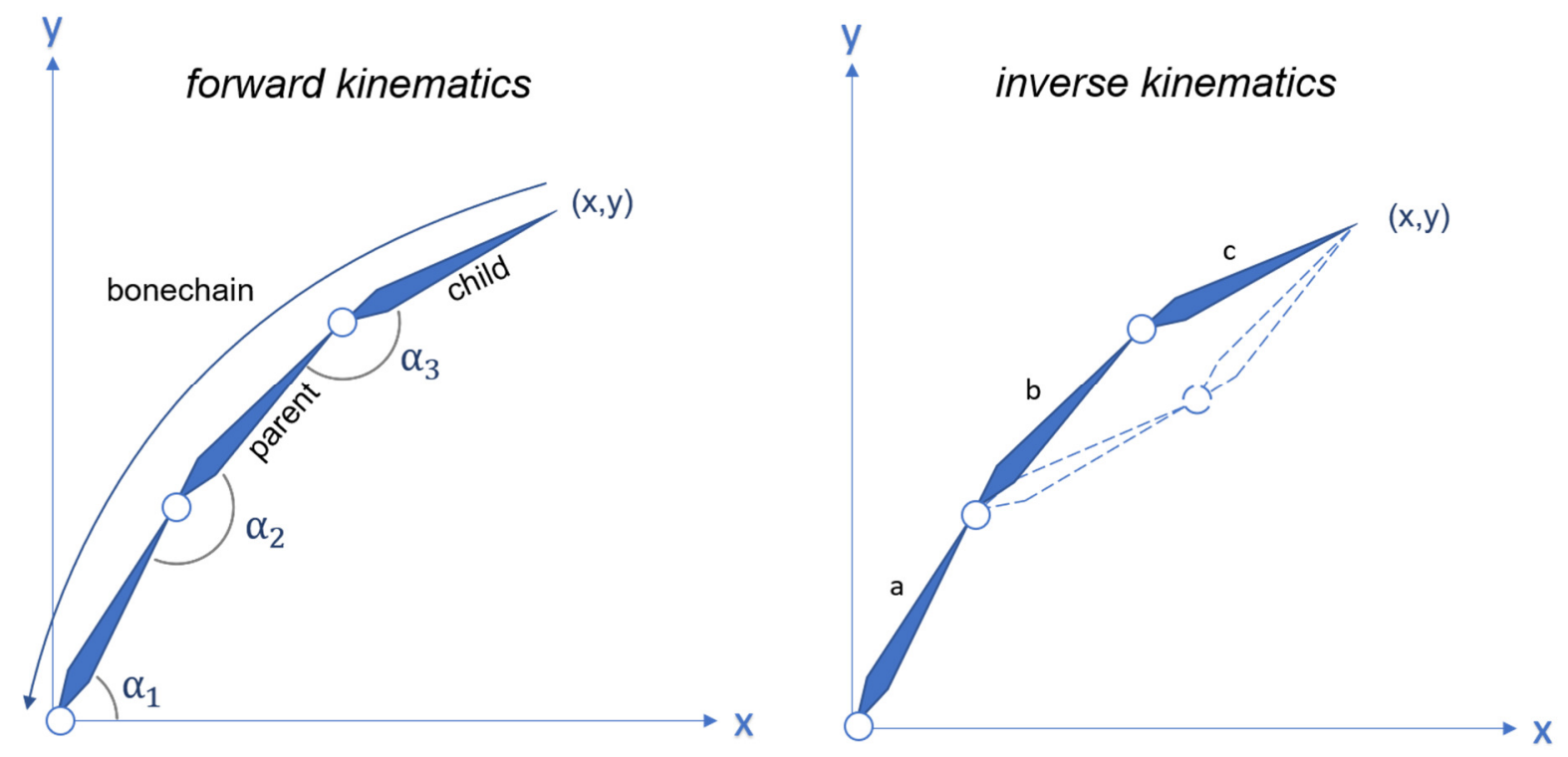

Figure 1: forward and inverse kinematics explained in three bones

The advantages of skeleton animations are a clear assignment of points, bones can be controlled individually. An animation can also be defined by simple movements of the bones instead of point by point. A big disadvantage of this animation technique is that a bone only defines a set of vertices. This means that realistic skin and muscle movements can only be shown to a limited extent. For this reason, special muscle controllers are needed, which correspond to the human anatomy and are moved by the rotation of the bones as in reality. However, the creation of a muscular skeleton means an increased additional effort, which does not guarantee a successful surface deformation during a movement. In addition, the manual post-processing of an animation is also associated with an increased effort. In addition, digitally generated animations can only be checked optically for the correctness of their surface transformation or later confirmed with scans. A correct surface representation is extremely important for the digital fit test. However, the system internal avatars of the clothing simulation programs still vary greatly when the size or especially the pose is changed. The method presented in this paper describes the technique of generating a movement using two scans, through which the rig can be animated and at the same time the muscular change can be displayed.

\section{Method}

For the test setup, a test person age 27 of size $40(92.3 \mathrm{~cm})$ and a figure type strong was scanned according to SizeGERMANY with the "Little Alice" scanner from 3DCopysystems in 3 different movements. Three poses were extracted: the reference pose "relaxed", a half T-pose and a step pose. To record the surface change and the transformation of the skeleton, the landmarks defined according to Morlock et al. (2018) were applied beforehand. ${ }^{[12]}$ Furthermore, the test person was scanned in the same poses for comparison with the Vitus Smart XXL.

On the scan with the reference pose "relaxed" a template mesh that was developed in the project has been applied. This grid structure is optimised by the positioning and location of the squares for resizing in the avatars. Furthermore, lines for the optical detection of the main body dimensions are marked on the surface.

A skeletal structure was created that depicts the most important joints. In addition, the maximum angle of rotation for all three axes of the bones was defined internally in Blender according to Krzywinski et al. (2014). ${ }^{[8]}$ The foot and wrist joints as well as the neck and hip can be positioned by inverse kinematics controller. The targets are shown as spheres (left side figure 2), these are positioned within the scan geometry and the pose is predefined. The rotation of the chain of bones is controlled by the arrows in front of the spheres and adjusted to the scan. The transformation of the remaining bones can be controlled by the rotation values in the forward kinematics.

The previously created half-T pose and step pose scans were imported into Blender as an .obj file after filling in the gaps in Geomagic Studio caused by movement in the scanner. 


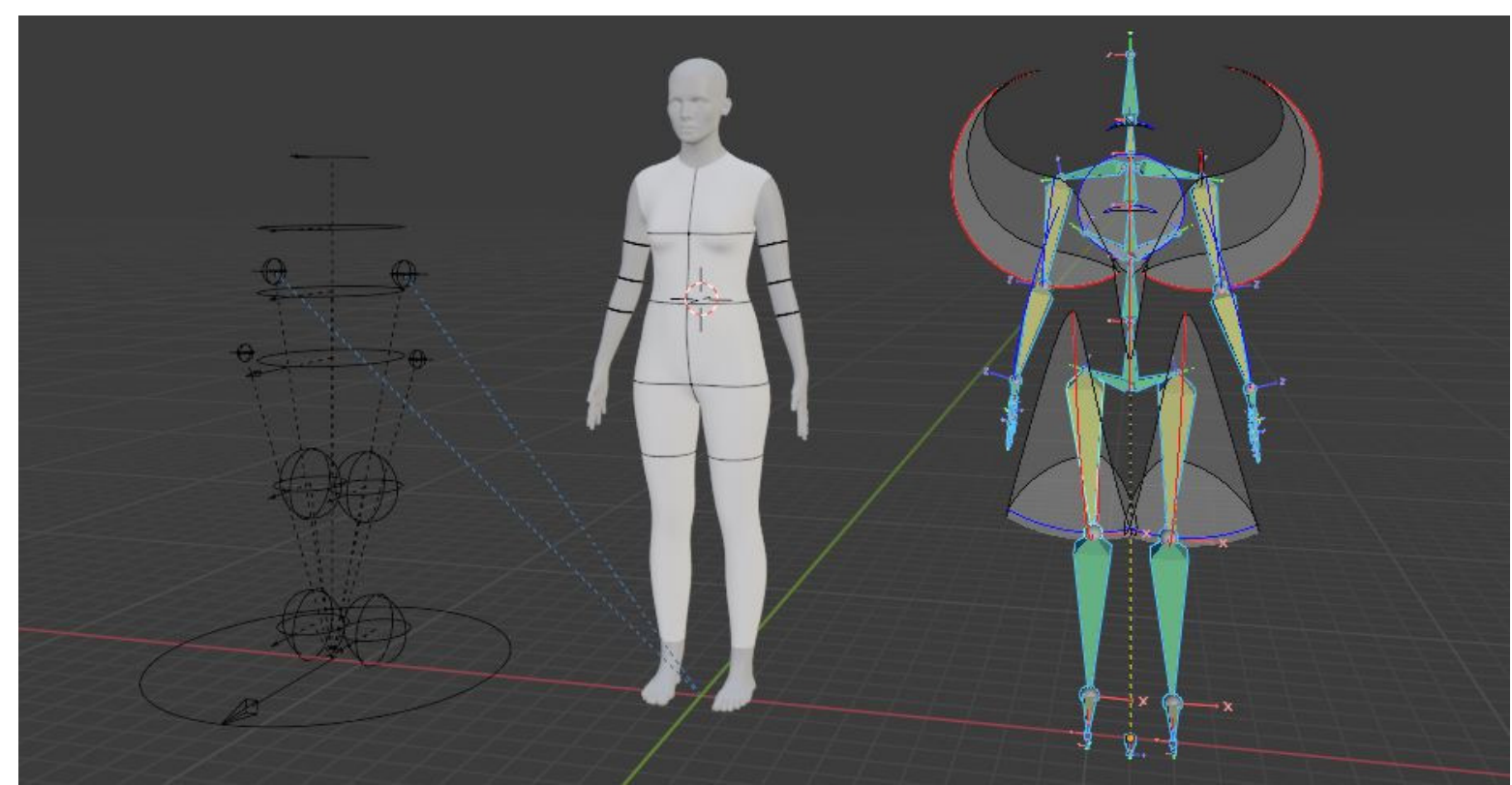

Figure 2: f.l.t.r inverse kinematics controllers, Template mesh with lines applied on the surface, rig with bone limitation surfaces

At first the digital image of the pose half $T$-pose was generated. After the positioning of the template mesh, the weights of the rigs were checked if they correspond to reality, otherwise they are adjusted. Points at critical locations, where the muscle movement cannot be represented by the rig, are collected in a new vertex group. These are then projected onto the scan geometry and represent a realistic surface. In the armpit area, the points that do not match the surface even after the weights have been adjusted are projected onto the scan geometry in the direction of their negative normal direction. Due to individual shadows underneath the arm, individual points may miss the previously determined position on the surface. These points must then be placed manually. Distortions and deformations of the polygon mesh caused by the projection are corrected with the smooth Laplacian algorithm. This corrected mesh is then saved as a shape key. A shape key stores one or more-point transformations that are relative or absolute to the base geometry. The corrected shape of a polygon mesh can thus be saved within a geometry and is thus very useful for fine-tuning the animation. In this case the mesh changes can be saved and animated in the rig animation. The saved information can be actively recalled and the influence on the base mesh can be precisely defined.

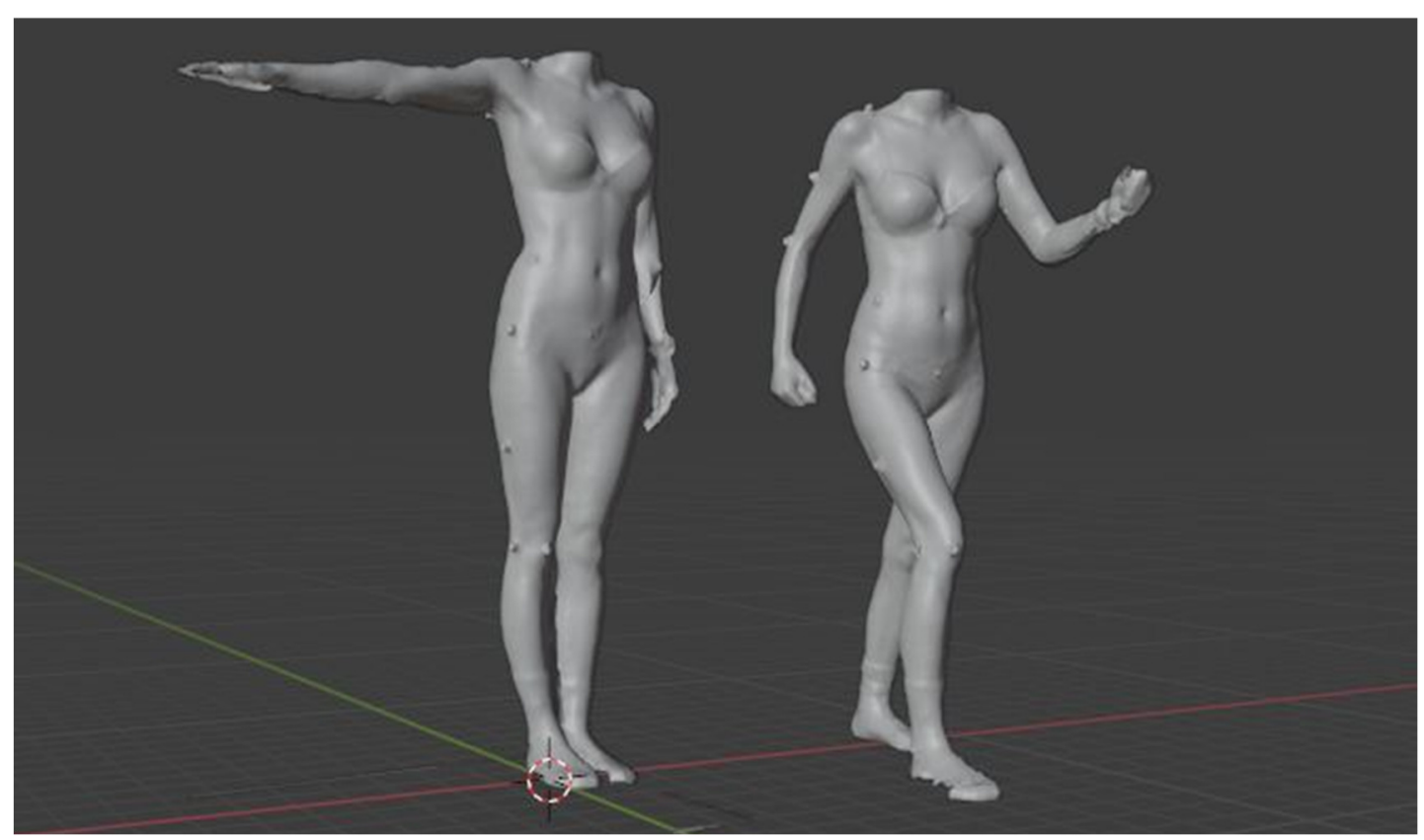

Figure 3: Scans half T-pose and step pose 
After the process of positioning and creating the shape keys for the pose half-T pose has been created. The transformation values of the inverse kinematics controllers are saved as a keyframe in frame 1.

In the next step the controllers will be positioned at the half step pose. A new keyframe will be created in which the transformation values of the controllers can be stored. Also, the vertex weights of the bones are checked and adjusted. Areas that do not reach the scan geometry through the rig and the vertex weights will be manually projected on the surface again and saved as a new shape key.

From the position and rotation data of the controllers stored in keyframe 1 and 30 , a motion curve can then be generated by interpolation. However, this movement curve is only valid for the controllers that control the rig. The movement of the bones can already be made visible via the motion paths. This curve shows the movement of the relevant bones and the position in the keyframe. Non-harmonic progressions give conclusions about an error in the preparation. For the exact motion data, the rotation and movement values for each bone of the rig are calculated and the dependence of the bones on the controllers is deleted.

The previously collected information about surface changes in the respective positions can be activated via the shape key editor, which is controlled by a function in the drivers editor. The surface change on the armpit is determined by the rotation around the x-axis of the upper arm. To activate this, the values of the angles on the upper arm calculated in the method are normalised to an interval of $[0,1]$ and this function is transferred to the shape keys. When this movement reaches frame 30 and thus the highest value of the $\mathrm{X}$ rotation, the shape key is fully activated.

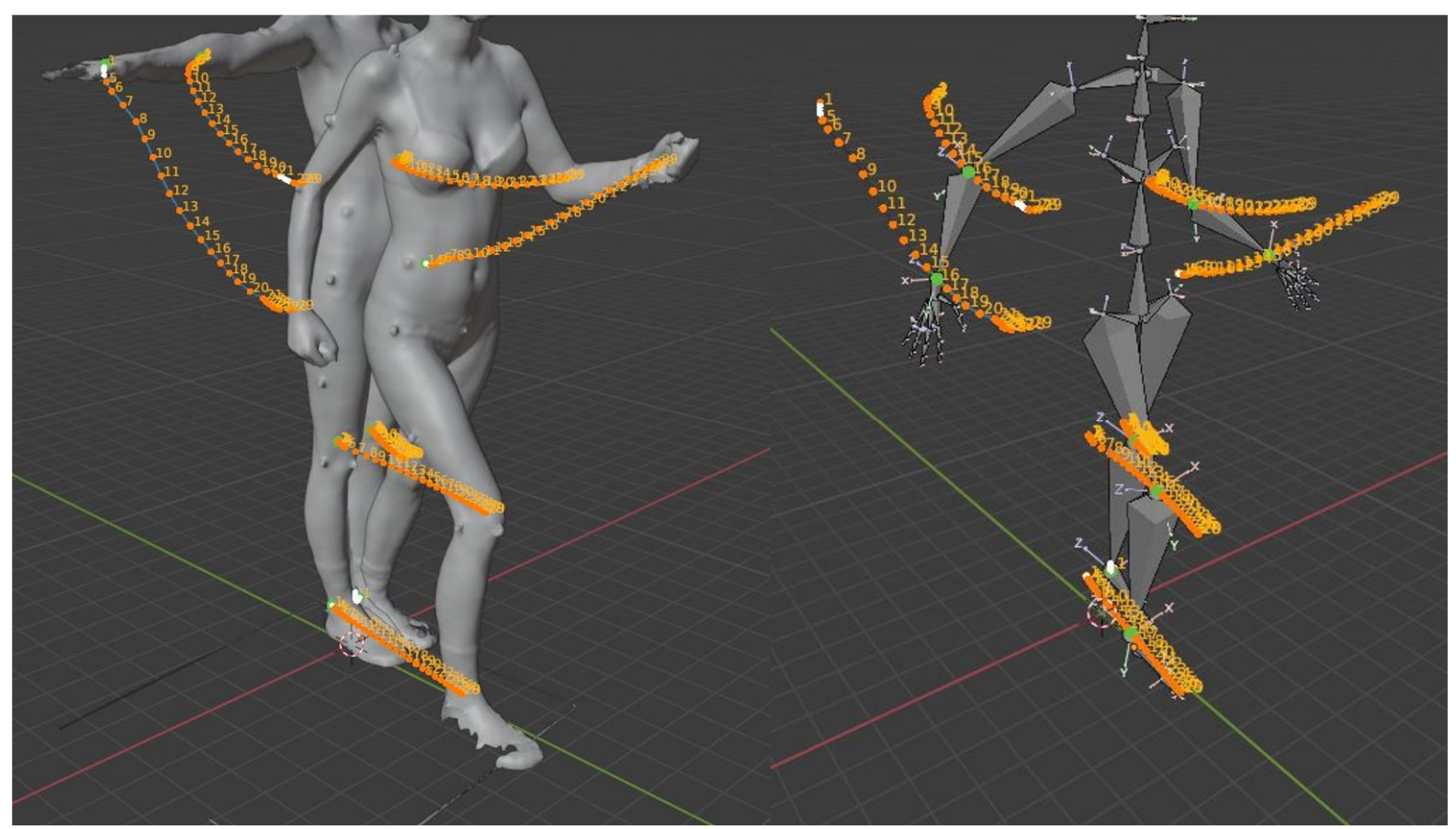

Figure 4: Motion paths with frame number

The markers on the surface are used to take circumferential measurements to validate the process and compare them with each other. First of all the lines A, B and C were chosen as reference. These markers also lie as texture on the generated template mesh. Between the defined points a line is generated along which contour curves are generated on the geometry. The length of the curves can then be compared. 


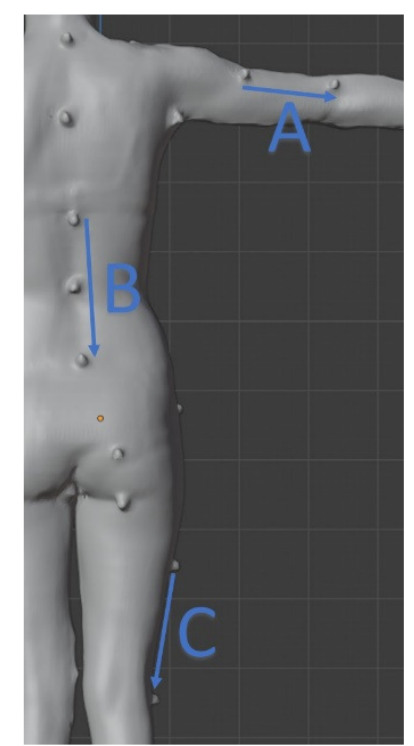

Figure 5: Defined measurement points

\section{Results}

In the project an new method was developed. This lead to overall better representation of human body shapes with avatars. Figure 6 shows in yellow, the deformation of the grid structure produced by the presented method and in dark colour the representation of the same grid structure without adjustment. It is clearly visible how differently the surface is deformed. Because the yellow surface is based on a scan, it can be said that it corresponds more to reality. Between the defined points a line is generated along which contour curves are generated on the geometry. The length of the curves can then be compared. The percentage deviation of the circumferential dimensions between scan and new contour is on average less than $2 \%$. This can be explained by the irregularity of the surface after scanning.

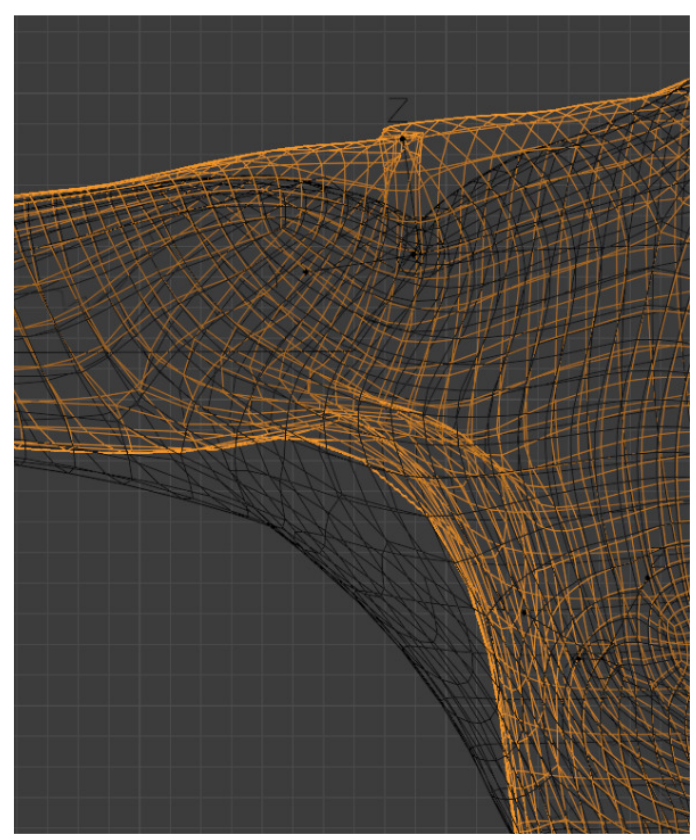

Figure 6: Difference between meshes

The generated animation data is stored in a blender internal library with the corresponding metadata of the test person. In addition to the animation data, the mesh information from the shapekeys is also saved.The resulting motion of the avatar will then be imported into the common used simulation softwares in the corresponding 3D exchange formats and used for a fit check in motion. 


\section{Conclusion}

Utilizing 3D simulation software to assess garment fit accurate avatars of the target group are the foundation. Current default system avatars still show limitation. Therefore, a new method to generate rigged avatars was developed. The movement generated by the rig, extended by the scans results in a more precise surface representation than that generated in the current state in the simulation programs. The cause of fitting problems can be investigated and repaired more easily. Another problem of fitting in motion is the self-intersecting of the surface in certain poses. The adjacent geometries around the joints overlap so much that a qualitative statement about the fit is not possible. Unfortunately, the interpolation of the targets is still linear now, this can lead to the motion path passing close to another bone. In upcoming studies, it will be investigating how the motion paths of the targets can be manipulated in a way that a self-penetration is not allowed. The created poses and changes on the surface caused by the angles of the bones to each other are collected in a library. The movements and poses can be freely combined with each other and adapted for every situation of a fit.

The data sets can, for example, be structured according to age or occupational group. In this way it is possible to influence which person performs each movement and how the speed and motion path of the movement changes as a result.

The circumference values measured in the method are an important part of the investigation of the change in body shape during movement. In future investigations the length differences will be measured and compared. These data can support the design of functional clothing and lead to better results. These can be tested immediately in the digital environment on the generated movement.

\section{References}

[1] S. Jevšnik, Z. Stjepanovič, A. Rudolf, "3D Virtual Prototyping of Garments: Approaches, Developments and Challenges", Journal of Fiber Bioengineering \& Informatics 2017, 10, 51-63.

[2] E. Lapkovska, I. Dabolina, "An investigation on the virtual prototyping validity - simulation of garment drape ", 4, 2018.

[3] J. Lee, Y. Nam, M. H. Cui, et al., in Secondary "Fit Evaluation of 3D Virtual Garment", (Ed.:^(Eds.: Springer Berlin Heidelberg, Berlin, Heidelberg, 2007, 550-558.

[4] H. K. Song, S. P. Ashdown, "Investigation of the Validity of 3-D Virtual Fitting for Pants", Clothing and Textiles Research Journal 2015, 33, 4, 314-330.

[5] https://www.linkedin.com/posts/clo-virtual-fashion-inc- clo-clo3d-itsclo3d-activity$6688790000223436801-c 7 u d, 10.09 .2020$.

[6] S. Morlock, J. Keinath, "Virtual Designing and Fitting - 3D Simulation in Clothing Development", Performance Days, München (D), 2019.

[7] A. S. M. Sayem, " Virtual fashion ID: a reality check", IFFTI Conference, 8 -11 April 2019, Manchester Fashion Institute, Manchester (GB), 2019.

[8] S. Krzywinski, E. Wendt, A. Leipner, et al., "Kinematische Menschmodelle zur Produktentwicklung von Bekleidung (Kinematische Menschmodelle)", IGF 17355 BG/1, Dresden, Hohenstein, 2014.

[9] S. Morlock, "Entwicklung einer neuen HAKA-Grundschnittkonstruktion zur Umsetzung der Funktionsmaße in bewegungsoptimierte Bekleidungsprodukte (unpublished Project Proposal)", IGF Nr. 19912N, Hohenstein Institut für Textilinnovation gGmbH, Hohenstein, 2017.

[10] A. Klepser, "Grundlagenuntersuchung zur Erschließung der 4D-BodyScanner-Technologie für die Analyse bekleidungsbedingter Mobilitätsrestriktionen (unpublished Project Proposal)", IGF Nr. 20163N, Hohenstein Institut für Textilinnovation gGmbH, Hohenstein, 2017.

[11] I. Baran, J. Popovic, "Automatic rigging and animation of 3D characters", ACM Transactions on Graphics (SIGGRAPH 2006),25(3) 2007, 26, 3,

[12] S. Morlock, C. Lörcher, A. Schenk, "Entwicklung eines ergonomisch- und bewegungsorientierten Größensystems für Funktionsmaße zur optimierten Gestaltung von Berufs- und Schutzbekleidung. Laufzeit 1.1.16 bis 30.06.18", IGF Nr. 18993N, Hohenstein Institut für Textilinnovation gGmbH, 2018. 